\title{
The Research on Key Technologies of Health Management of Modern Airliner Flight Control System
}

\author{
Duanzhaobin ${ }^{1, \text { a }}$ \\ ${ }^{1}$ Engineering and Technology Training Center, Civil Aviation University of China, \\ Tianjin, 300300 \\ a26428496@qq.com
}

Key words: Airliner; Airliner Flight Control System; Health management; Data collection; Fault prediction

\begin{abstract}
With the increase of people's dependence on aircraft, the safety of aircraft has become one of the focuses. The introduction of health management technology to increase the modern airliner control system management, as well as the aircraft flight data acquisition, fault prediction and fault diagnosis for security have become the main trend, which can further improve the reliability of civil aircraft flight system, effectively reducing the probability of failure of the aircraft, and providing technical support to increase the safety of modern airliner.
\end{abstract}

\section{Overview of Plane Health Management System}

As the aircraft system becomes more integrated and complicated, new methods have been developed in 70s to monitor system status, such as BIT, fault protection and redundancy management. Subsequently, the technology that diagnose the source and cause of fault on the aircraft emerges, which finally led to the birth of the fault prediction method. In the project development stage, PHM begins to be put forward, which is the development of the aircraft BIT and monitoring in use. This development is to change from the health monitoring and health management, which introduces the fault prediction ability. This ability can be used to identifying and managing the fault from the angle of the whole system. Its purpose is to reduce maintenance manpower and realize autonomous guarantee.

PHM technology has attracted more and more attention by the military and industry. But as an emerging discipline, it has not yet put forward a complete system architecture, its function and concept is still in the verification phase. Especially the fault prediction technology,which is not yet mature and not into the application, but it represents an important development trend of PHM in the future. At present, the biggest obstacle to PHM technology is the uncertainty of the remaining life cycle prediction and the detection of the intermittent failure of electronic products. The development of PHM technology in our country is still at the stage of theoretical research and primary application. The key technologies involved in the airliner health management includes technology, diagnosis technology, forecast technology and 
management decision-making technology acquisition. They are important parts of PHM technology and provide powerful technical support for the safety of modern airliner.

Diagram 1 The Diagram of Development Course of PHM Technology

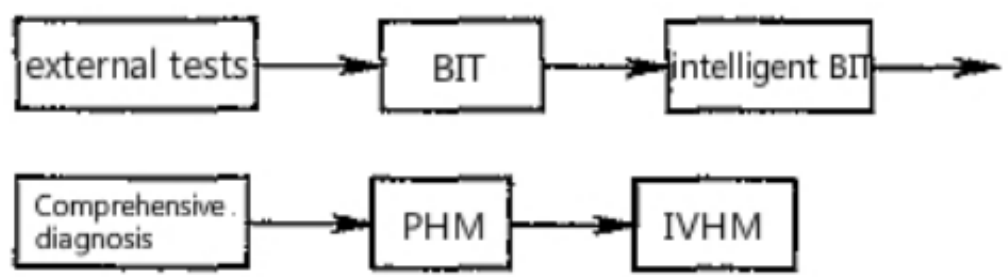

\section{Application of Key Technologies of Health Management in Flight control system of modern civil aircraft}

As the actual flight test results show, after the airliner uses the flight control system, the fuel consumption can be saved by 2\%-5\%. The common Airliner flight control system consists of Flight Management Computer System(FMCS), Autopilot and Flight Director (AP/FD), Thrust Management System (TMS), Inertial Reference System (IRS), Electronic Fight Instrument System (EFIS), Air Data Computer (ADC); Instrument / Microwave landing System (ILS/MLS), Air Collision Avoidance and Warning System, Engine Indicating and Crew Alerting System (EICAS), and Distance Measuring Equipment/Very-high-frequency Omnidirectional Range(DME/VOR).

\section{Application of flight data acquisition technology in modern civil aircraft}

The data acquisition system on the aircraft will gather and transfer the signals received by sensors, according to the order and time intervals, to the airborne data acquisition and recording device, so that some of the parameters can be easily handled on the plane, some faults can be identified and then display to the drivers through a variety of ways (lighting, alarm, display panel to display etc.) . Almost all of these data will be transmitted to the ground analysis system for more sophisticated and complex data processing. Analyzed by models and systems such as state monitoring, fault identification, fault prediction, these results will be fed back to the relevant departments in charts, report, trend charts and other forms to provide improved aircraft design, use, maintenance, and decision making.

The various systems and sensors of the aircraft produce various signals. Generally, signal types that input to the flight data acquisition unit (FDAU/DFDAU) include analog signal, discrete signal and digital signal. Sometimes the discrete signal is classified into analog signals and called switch analog signals, while analog signals traditionally is called a continuous quantity analog signal. For analog signals, a port generally corresponds to one parameter, and the sensor line is directly connected to the acquisition component. Analog signal analog signal is divided into continuous analog signal and digital signal sensor switch. For digital signal, the sensor is firstly connected to functional unit (such as air data computer), then connected to data acquisition module by ARINC429 data bus. One port corresponds to multiple 
parameters, commonly known as the ARINC429 bus or DITS argument. The design specification of the collecting component defines all the input parameters.

If the Bits1-8 of one ARINC parameter is 01101111 which can be converted to octal $(01101111)_{2}=(157)_{8}$, then the parameter number is 157 . Each data word of DFDR or QAR is 12-bits, corresponding to some bits in the Bitsll-28 of ARINC429. The specific bits corresponding is clearly specified in the relative manual.

The collected flight data are recorded in different types of recorders, including DFDR, QAR, WQAR, and DAR. The records of DFDR, QAR, or DAR generally follow the ARINC717 specification, whose data is on a pair of twisted pairs, and data loops are recorded one frame at a time. Each frame of data is 4 seconds, and the data per second is also called a sub frame. Each sub frame generally is divided into 64 characters, 128 words, 256 words, 512 words or 1024 words, and each word has 12 data bits .

The so-called decoding, in fact, is a process that convert the original data in binary arrangement recorded in a DFDR or QAR or DAR recorder into visual engineering data with unit. It is the inverse process of records, and the key point is to clarify the mapping relation of ARINC717 specification.

\section{Application of Fault Prediction Technology for Modern Airliner}

The prediction is to analyze the data through the establishing a model, or assessing presumable characteristic value or range of values of unlabeled samples. The prediction is summarizing and describing the trend of upcoming events or data. Though the analysis and research on the prediction of aircraft fault by researchers at home and abroad, some effective methods have been put forward, which are some general techniques for aircraft fault prediction, including regression analysis, time series analysis, gray model analysis, neural network and support vector machine method and so on.

\section{Regression Analysis}

The regression analysis is to establish a regression analysis model from the correlation between the predicted object and its dependent variable, and a quantitative method used to predicting the future development trend of the object. As to the objects with no clear correlation, although this method cannot directly measure their relationship, through a lot of data it can dig out the statistical regularity between them. The regression analysis is to establish correlation function between the objects and describe the correlation between the objects by finding out the inherent laws of statistics. Then though the expression of correlation function possible outcome of predicted objects can be predicted, which can be used for effectively preventing and controlling the development of the objects.

\section{Linear Regression Model}

The simplest linear regression model, such as equation (1), in which $\alpha$ and $\beta$ are called regression coefficients 
$Y=\alpha+\beta x$

\section{Least Square Fitting}

In the equation (1),regression coefficients $\alpha$ and $\beta$ can be obtained by observing $\left(X_{\mathrm{i}}, Y_{\mathrm{i}}\right)(\mathrm{i}=1,2, \ldots, \mathrm{n})$, and observation pairs can be obtained by experiment. The question is to approximate the result of calculation with the value of $Y_{i}$ and to find the parameters that satisfy this requirement. However, in practice, there is a certain error between the observed value and the actual value. If the error value is $E_{i}$, then the random variable $E_{i}$ should be satisfied:

$$
E\left(E_{\mathrm{i}}\right)=0, \operatorname{cov}\left(E_{\mathrm{i}}, E_{\mathrm{j}}\right)=0
$$

The least square takes the minimum variance of the random variable as the error criterion and calculates the regression coefficients $\alpha$ and $\beta$.

$$
\sum_{\mathrm{i}=1}^{\mathrm{n}}\left(Y_{\mathrm{i}}-\alpha-\beta X_{\mathrm{i}}\right)^{2}
$$

Compute the minimum, when taking the extreme value

$$
\frac{\partial\left[\sum_{\mathrm{i}=1}^{\mathrm{n}}\left(Y_{\mathrm{i}}-\alpha-\beta X_{\mathrm{i}}\right)^{2}\right]}{\partial \alpha}=0 \frac{\partial\left[\sum_{\mathrm{i}=1}^{\mathrm{n}}\left(Y_{\mathrm{i}}-\alpha-\beta X_{\mathrm{i}}\right)^{2}\right]}{\partial \beta}=0
$$

Solving simultaneously:

$$
\begin{gathered}
\alpha=\bar{Y}-\beta \bar{X}, \quad \beta=\frac{\sum_{\mathrm{i}=1}^{\mathrm{n}}\left[\left(X_{\mathrm{i}}-\bar{X}\right)\left(Y_{\mathrm{i}}-\bar{Y}\right)\right]}{\sum_{\mathrm{i}=1}^{\mathrm{n}}\left(X_{\mathrm{i}}-\bar{X}\right)^{2}} \\
\bar{X}=\frac{\sum_{\mathrm{i}=1}^{\mathrm{n}} X_{\mathrm{i}}}{\mathrm{n}}, \quad \bar{Y}=\frac{\sum_{\mathrm{i}=1}^{\mathrm{n}} Y_{\mathrm{i}}}{\mathrm{n}}
\end{gathered}
$$

\section{Support for Fault Prediction and Fault Diagnosis Based on Flight Data Decoding and Flight Data}

The decoding and application of flight data play a very important role in the whole system. The decoding of flight data realize the decoding of the original flight data recorded by QAR and FDR into the engineering value of the flight parameters, which is the basis for the monitoring of the various aircraft systems and components. The support methods of flight data to fault prediction and fault diagnosis include flight 
parameter query, cockpit display, flight parameter change curve, takeoff and landing 3D simulation and overrun report. These means use the decoded engineering parameters as the data source, realizing the operation quality analysis and monitoring of aircraft systems and components. Consequently, according to the results of monitoring and analysis, potential faults and faults that have already happened will be timely found, providing substantial fault information based on the case reasoning system, and the support of fault prediction and diagnosis

The design first needs to carry on the flight parameter database, various data tables based on the needs of the decoding and application of flight data as shown in Diagram 3 and Diagram 4.

Diagram 3 The Structure and Relation Diagram of Flight Data Decoding Correlation Table

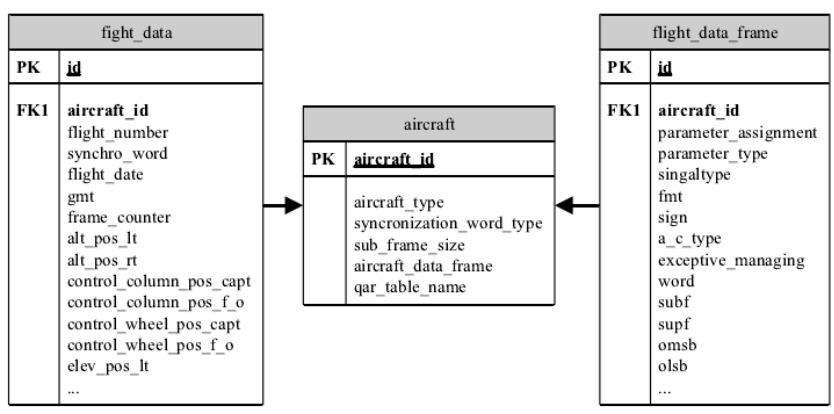

Diagram 4 The Structure and Relation Diagram of Flight Parameter Application Table

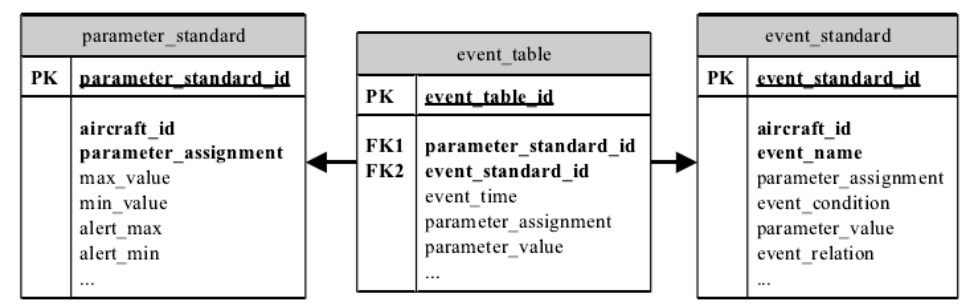

\section{Conclusion}

With the support of health management, we can strengthen the the acquisition and processing, fault diagnosis, prediction and assessment of operation information of every part and component of modern airliner flight control system, and finally send out the corresponding alarm or message to the users and ground monitoring room with health status reports, sound or light. It will accurately estimate the fault plane, reduce the probability of failure, provide technical support for the safety of the aircraft, and has a certain practical significance.

\section{Acknowledgement}

The Fundamental Research Funds for the Central Universities, Specialized Fund for Civil Aviation University of China（3122016D006） 


\section{Reference}

[1]Liu Chao.The Research on Modern Civil Aircraft Health Management Technology[J].Science and Technology Information, 2014,12(19): 61

[2]Cao Quanxin, Yangrong, Liu Ziyao.The Research on Health Management Technology of Civil Aircraft[J].Avionics, 2014,45(4): 15-19

[3]Zhang Xinbo.The Research on Key Technologies of Flight Test Evaluation for Civil Aircraft Flight Management System[D].Engineering Master's Thesis of Xi'an Electronic and Science University, 2013

[4]Liu Jianwei, Jiang Bin.The Research and System Development of Health Monitoring Technology for Flight Control System[J].bulletin of science and technology, 2011,27(5): 787-791

[5]Gong Sifang.The Research on Aircraft Fault Prediction Model and Method Based on Data Mining[D].Master's Thesis of Xi'an Electronic and Science University, 2015

[6]Qin Liyong.The Research on Aircraft Fault Prediction and Fault diagnosis System Based on Flight Data[D].Master's Thesis of Nanjing University of Aeronautics \& Astronautics, 2007

[7]Wang Chen.The Design of Fault Diagnosis Expert System for Aircraft Automatic Flight System[D].Master's Thesis of Nanjing University of Aeronautics \& Astronautics, 2015

[8]Li Luyao.The Research on Civil Aircraft Flight Control System Fault Prediction Technology[D].Master's Thesis of Civil Aviation University of China, 2014 